\title{
Survey Of Male And Female Frogs (Pyxicephalus Edulis) And Assosciated Toxic And Non Toxic Elements
}

Thompson Faraday Ediagbonya ( $\nabla$ tf.ediagbonya@gmail.com )

Ondo State University of Science and Technology https://orcid.org/0000-0003-0878-8851

Adedayo Johnson Ogunjobi

Olusegun Agagu University of Science and Technology

Ademola Charles Adenikinju

Olusegun Agagun University of Science and Technology

\section{Research Article}

Keywords: Male frog, female frog, element, Pyxicephalus edulis, sediment, PIXE

Posted Date: June 2nd, 2021

DOI: https://doi.org/10.21203/rs.3.rs-581624/v1

License: () (i) This work is licensed under a Creative Commons Attribution 4.0 International License. Read Full License 


\section{Abstract}

The present research was performed to determine the concentration of heavy metals in the male and female specimens of the identified frog species: pyxicephalus edulis collected from the Igbekebo River in Igbekebo, Ese-odo local government. Adult frogs (male and female) were collected from the river bank, and sediment samples and water samples were also collected at five ( 5) separate locations in the river. The frogs were dried separately at $105^{0} \mathrm{C}$ for 6 hrs and then crushed into small particles (powder form). The sediment samples were air-dried for three days. Elemental components in frog samples and sediment samples were analyzed using Proton Induced X-Ray Emission (PIXE). Physiochemical parameters and heavy metals of the water samples were also analyzed. The findings showed that the concentrations of $\mathrm{Si}, \mathrm{P}, \mathrm{Cl}, \mathrm{Ni}, \mathrm{Zn}$ and $\mathrm{Cd}$ were higher in Male frog while $\mathrm{Mg}, \mathrm{Al}, \mathrm{K}, \mathrm{Ca}, \mathrm{Ti}, \mathrm{V}, \mathrm{Cr}, \mathrm{Mn}, \mathrm{Fe}, \mathrm{Co}, \mathrm{Cu}, \mathrm{Zr}, \mathrm{Pb}$ and Sn were higher in female frogs, the explanation for this variability is not known but may be due to variations in the genetic make-up of Male and Female frogs. The concentration of heavy metals in both male and female frogs was substantially higher relative to the available WHO limits. The mean concentration of elemental constituents in sediment was higher than the IAEA limit. The values of enrichment and the Igeo values were very high.

\section{Introduction}

The worldwide decline in amphibian species has shown over-exploitation as one explanation for a decrease in global amphibian populations (Stuart et al., 2008). It has been said by Niasse et al ., (2004) that 281 amphibian species have the primary threat of utilization, $54 \%$ of which are already listed in their categories as vulnerable, endangered or critically endangered. A recent research by Warketin et al. (2009) sums up troubling data on Asian frog collected for use, with Mohneke (2011) reporting that a total of two million, 7800 and 610 frog species have been collected annually by 302 frog collectors in the southwest countries of Nigeria.A food scarcity and Africa behind other developed countries is one of the most apparent consequences of the world's population growth (Lameed, 2008).As a result, the production and supply of animal protein for feeding ever-expanding population decreased significantly (Akpan et al ., (2009). Frogs have often been collected on a local scale as an important source of animal protein (Angulo, 2008; Mohneke et al. 2009) in a large number of African countries (such as Benin, Burkina Faso, Cameroon, the Ivory Coast (Ghana), Guinea, Namibia, Nigeria, South Africa and Togo).An increase in human population level continues to raise the current burden on wildlife. Frog species are generally extracted from wild animals in various areas of the world primarily for fruit, medicinal purposes and pet trade (Mohneke et al., 2011). Agriculture of these used frog species has traditionally been ignored in Africa and the world as a whole, although the rate of consumption continues to increase. Most frogs in advanced countries come from developing countries. Therefore, the rate of exports of frogs from developing countries (regardless of their conservation status) to developed countries has increased due to a high demand market. Onadeko et al. ( 2011) stated that frog legs were common in Europe and even eaten in countries where hunting frogs is prohibited legally. Frogs are called 'jumping chickens' in many regions in Asia (Indonesia) and Africa (Nigeria) as a taste similar to chicken (Altherr et al. 2011). Its availability, the taste for people and the need to meet the demand for protein are some of the key reasons for the annual consumption of billions of frogs. Leather and souvenirs, pet trades and culture, including traditional medicine are also collected for the purpose of collection (Kusrini and Alford, 2006; Gonwouo and Rodel, 2008). Overuse of these tools is, however, one of the main biodiversity challenges. Frogs became a trading commodity for more than 30 countries in the last decade, with trade in frog species worth about US\$ 48.7 million in 1998 (Teixeira et al., 2001).Pollution of heavy metals is harmful to our environment (Hazrat et al 2019). All of these elements are poisonous to human and animal microorganisms, they are necessary, not important. Excess heavy metal deposition in the body could result in vomiting, vision loss, thyroid, asthma, abdominal pain, sore throat, lung cancer, nose cancer, dizziness, and human heart problems (Tchounwou et al 2012).The aims of this study were to identify male and female frogs' species and to assess the elemental composition of male and female frogs as well as elemental composition and the physicochemical parameters of the sediment in the study area of Igbekebo. Relatively few studies in amphibian species in natural populations have been carried out concerning heavy metal accumulation (Sura et al. 2006). The Rana ridibunda Frog was used as a heavy metal contamination bioindicator (Efstathiadou et al. 2007; Fenoglio et al. 2011; Loumbourdis et al. 2007; Simon et al. 2010).Amphibian species have been suggested as an important environmental indicator of pollution for the frog limb as being more sensitive than other aquatic vertebrate bioindicators, due to the fact that they have a permeable skin that is able ready to absorb contaminants from the atmosphere (Schuytema and Nebeker 1996; Simon et al. 2012)

\section{Materials And Method}

\section{Study Area}

In Ondo State, Nigeria, Igbekebo is headquartered in the Local Government Area. It is populated by the Western Apoi and Arogbo ethnic subgroups ljaw (Izon). At the 2006 census, the population is 762 square kilometers and 154,978. Because of its fishing and lumbering activities, the Igbekebo river has a high financial influence. Due to the chemical and biological inputs in the river the river is highly contaminated and this can result in high concentrations of heavy metals. Igbekebo is an area of oil production that is very polluted by petroleum spillage due to bunkering in the field. It is an urban small rural village. Figure 1 indicates the various locations for sampling.

\section{Sampling}

Two adult frogs (male and female) were collected from the river bank and sediment samples were collected at five (5) different locations in the river. The samples of the frogs were labeled as samples A and B i.e. the male frog was labeled as sample A while the female frog was labeled as sample B, the sediment samples were labeled as location A, B, C, D, E.

\section{Sample Preparation}

The frogs were oven dried at $105^{\circ} \mathrm{C}$ separately for 6 hours then crushed and sent for analysis in small particles (powdery form). The sediment samples were air dried for three days and sent for analysis, and the water samples were also sent for analysis.

Metal analysis of some physio-chemical parameters of sediment Samples 
Analysis was carried out with a 1.7 MeV 5SDH Pelletron Accelerator for sample metal concentrations. The pellets of sample were then analyzed for the total content of the elements (expressed as $\mathrm{mg} / \mathrm{kg}$ ) by the Particle-Induced X-ray Emission (PIXE) technique (He et al., 1993; Johansson et al., 1995) The technical detection limit is from 0.1 to $10 \mathrm{mg} / \mathrm{kg}$. Table pH and conductivity (Hanna 991300) meters have been used to measure the pH and electric conductivity of the ground sample. The nitrate and chloride were also determined using standard procedures (Ademoroti 1996).Detailed analyses had been reported by

(Ediagbonya and Balogun ,2020a;Ediagbonya et al 2020)

\section{Bioaccumulation factor}

Bioaccumulation factor (BAF) explains the intake and distribution of the element in the organism after exposure in a given environmental matrix (Subotić et al., 2013); and has been used here to determine the level of frog with a higher risk to health (DeForest et al., 2007). The concentration of the sediment elements in the frog was measured as a ratio between the concentration of the frog element and the concentration in sediment (Liao and Ling, 2003; Javed and Usmani, 2013) and Bio-accumulation factor (BAF). BAF $<1$ indicates no contamination of the frog; $1>B A F \leq 10$, the frog is tolerant and BAF $>10$, a hyperaccumulator (Ávila et al., 2017).

\section{$\mathrm{BAF}=C($ Frog $) / C$ (sediment $)$}

\section{Health Risk Assessment}

Risk was assessed using the target hazard quotient (THQ) and the hazard index ( $\mathrm{HI})$. The risk to human health derived from the ingestion of contaminated food as specified by FAO / WHO (2010) and the target hazard quotient (THQ) as set out in the USEPA Region III Risk-based Concentration Table (USEPA, 2015). The THQ was calculated using the formula given by (Singh et al., 2010)THQ $=\mathrm{Efr} \times \mathrm{ED} \times \mathrm{FIR} \times \mathrm{C} / \mathrm{RfDo} \times \mathrm{Baverage} \mathrm{wt} \times \mathrm{ATn} \times 10^{-3}$

Efr is exposure frequency assumed to be 365 days year - 1, ED is exposure duration in 54 years an average lifetime for Nigeria, while the average body weight used was $65 \mathrm{~kg}$ for this study (Oguntona,1998) FIR is average daily consumption taken as $1.95 \times 10^{-2} \mathrm{~kg} \mathrm{person}^{-1} \mathrm{day}^{-1} \mathrm{C}$ is concentration of metal in frog sample in mg/kg, RfDo i is the oral reference dose ( $\mathrm{Al}, \mathrm{Ti}, \mathrm{V}, \mathrm{Cr}, \mathrm{Mn}, \mathrm{Co}, \mathrm{Pb}, \mathrm{Cd}, \mathrm{Cu}, \mathrm{Zn}, \mathrm{Fe}$ and Ni (USEPA,2011,2013 ;RAIS, 2017); and ATn is average exposure time for non-carcinogens and is taken as 19710 days. The hazard index $(\mathrm{HI})$ from the consumption of frog estimated as the sum of THQs of all the metals in the frog and was expressed as follows;

$\mathrm{HI}=\mathrm{THQAl}+\mathrm{THQTi}+\mathrm{THQV}+\mathrm{THQCr}+\mathrm{THQMn}+\mathrm{THQFe}+\mathrm{THQCo}+\mathrm{THQNi}+\mathrm{THQCu}+\mathrm{THQZn}+\mathrm{THQCd}+\mathrm{THQPb}$

\section{Geo- Accumulation Index (Igeo).}

The Geo Accumulation (Igeo) index is intended to measure the extent of sediment pollution as calculated by (Loska et al.1997;Müller, 1979). It was used by $\operatorname{lgeo}=\ln [\mathrm{Cn} / 1.5 \times B n]$ (Ediagbonya and Ayedun,2018; Ediagbonya and Balogun,2020). The calculated metal ' $n$ ' content in sediments is $\mathrm{Cn}$ and the background concentration of the same metal is $\mathrm{Bn}$.

Enrichment Factor (EF)

To assess the extent of pollution in soil and sediments, the enrichment factor (EF) is used to determine excessive metal concentrations in sediments and soil. Al and Si were used as reference elements in this analysis. Few authors have used the reference feature of these components (Schiff and Weisberg, 199; Ediagbonya et al 2020b). The metal EF is classified as follows, according to Ergin et al., (1991):

$$
E F=\frac{\left(\frac{X}{A l}\right)_{\text {sediment }}}{\left(\frac{X}{A l}\right)_{\text {crust }}}
$$

Where $\mathrm{X} / \mathrm{Al}$ is the heavy metal concentration ratio $\mathrm{X})$ to the concentration of Al.

Wedephol was taken from the reference crustal ratio of the shale value or lithology (1968)

\section{Statistical data analysis}

For the statistical analyses in this report, IBM Statistical Package for Social Sciences (SPSS) version 24.0 was used. Descriptive statistics at the various sampling sites, such as range, mean, standard deviation for the psychochemical parameters as well as heavy metals. One direction Variance Analysis was used to conduct the spatial variation of heavy metal means at the various sample sites where substantial difference was found to distinguish significant means was used by the Duncan Multiple Range Test (DMRT). The physicochemical parameters, using the Pearson correlation, were also associated with the toxic elements. The multivariate analysis conducted for the source detection of heavy metals was the principal component analysis. The significance standard was set at $p<0.055$.

\section{Results And Discussion}


Table 1

Descriptive statistics of physiochemical parameters of soil samples in the sampled location

\begin{tabular}{|c|c|c|c|c|c|c|}
\hline & A & B & C & D & $E$ & $p$ \\
\hline Chloride & $10.67 \pm 0.78$ & $9.68 \pm 0.14$ & $10.90 \pm 0.17$ & $9.68 \pm 0.14$ & $10.90 \pm 0.17$ & 0.043 \\
\hline $\mathrm{pH}$ & $7.55 \pm 0.21$ & $7.70 \pm 0.14$ & $5.55 \pm 0.21$ & $7.70 \pm 0.14$ & $5.55 \pm 0.21$ & 0.000 \\
\hline$\mu s c m$ & $888.50 \pm 1.41$ & $934.30 \pm 2.12$ & $1120.20 \pm 0.71$ & $934.30 \pm 2.12$ & $1120.20 \pm 0.71$ & 0.000 \\
\hline Milligram/litre & $586.30 \pm 2.83$ & $613.20 \pm 1.41$ & $728.10 \pm 2.12$ & $613.20 \pm 1.41$ & $728.10 \pm 2.12$ & 0.000 \\
\hline Phosphate & $8.71 \pm 0.14$ & $5.28 \pm 0.14$ & $5.12 \pm 0.14$ & $5.28 \pm 0.14$ & $5.12 \pm 0.14$ & 0.000 \\
\hline Nitrate & $6.46 \pm 0.14$ & $6.52 \pm 0.14$ & $5.25 \pm 0.21$ & $6.52 \pm 0.14$ & $5.25 \pm 0.21$ & 0.001 \\
\hline Sulphate & $19.13 \pm 0.03$ & $19.73 \pm 0.03$ & $16.94 \pm 0.04$ & $19.73 \pm 0.03$ & $16.94 \pm 0.04$ & 0.000 \\
\hline
\end{tabular}

Table 1 shows the descriptive statistics on the physiochemical properties of soil samples at different locations. The highest mean chloride was recorded at locations $C$ and E, i.e. $10.90 \pm 0.17$ (10.75-11.09) and the lower mean chloride was recorded at locations B and D 7.70 \pm 0.14 (7.58-7.86) while the lower mean chloride was recorded at locations $C$ and E $5.55 \pm 0.21$ (5.36-5.76). The highest mean electrical conductivity was recorded at locations $C$ and $E 1120.20$ $\pm 0.71(1119.50-1120.93 \mu \mathrm{scm})$ and the lowest mean $\mu \mathrm{scm}$ was recorded at location $\mathrm{C}$ and $\mathrm{E} 1120.20 \pm 0.71$ (1119.50-1120.93 $\mu \mathrm{scm})$. Maximum mean was obtained at locations C and E $728.10 \pm 2.12$ (725.98-730.24 mg / I), while the smallest mean $\mathrm{mg} / \mathrm{I}$ was shown at location A, 586.30 \pm 2.83 (583.49-589.15). The maximum mean of phosphate was seen at location $A, 8.71 \pm 0.14(8.59-8.37)$, while the smallest mean of phosphate was recorded at locations $C$ and $E$, $5.12 \pm 0.14$ (5.00-5.28). The maximum mean of nitrate was reported at locations $B$ and D, $6.52 \pm 0.14(6.40-6.68)$, while the smallest mean nitrate was recorded at locations $C$ and $E 5.25 \pm 0.21(5.06-5.46)$. The maximum of mean sulphate was recorded at locations $B$ and $S 19.73 \pm 0.03(19.90-19.78)$, while the smallest mean of sulphate was recorded at locations $C$ and $E, 16.94 \pm 0.04$ (16.90-17.00) 
Table 2

Mean of the elemental concentrations in sediment in samples A-E (mg kg-1)

\begin{tabular}{|c|c|c|c|c|c|c|}
\hline & A & B & $\mathrm{C}$ & D & $E$ & $p$ \\
\hline $\mathrm{Na}$ & $4883.30 \pm 7.07$ & $4551.20 \pm 7.07$ & $5667.00 \pm 0.71$ & $6550.90 \pm 7.78$ & $4764.70 \pm 2.12$ & 0.000 \\
\hline$M g$ & $23227.60 \pm 7.07$ & $22752.60 \pm 9.90$ & $24124.80 \pm 2.83$ & $22950.60 \pm 3.54$ & $25127.60 \pm 7.07$ & 0.000 \\
\hline Al & $26427.20 \pm 5.66$ & $121552.80 \pm 7.07$ & $24291.00 \pm 4.24$ & $73994.50 \pm 67264.66$ & $24291.00 \pm 4.24$ & 0.076 \\
\hline $\mathrm{Si}$ & $346361.80 \pm 1.41$ & $300682.70 \pm 14.14$ & $245744.90 \pm 45.25$ & $323527.75 \pm 32293.64$ & $245744.90 \pm 45.25$ & 0.003 \\
\hline$P$ & $460.40 \pm 4.95$ & $117.90 \pm 7.07$ & $339.40 \pm 3.54$ & $284.90 \pm 243.24$ & $339.40 \pm 3.54$ & 0.161 \\
\hline $\mathrm{Cl}$ & $271.30 \pm 3.54$ & $88.00 \pm 2.12$ & $135.30 \pm 3.54$ & $180.15 \pm 132.44$ & $135.30 \pm 3.54$ & 0.150 \\
\hline K & $1464.60 \pm 18.38$ & $5222.40 \pm 7.78$ & $1789.80 \pm 4.24$ & $3347.25 \pm 2644.08$ & $1789.80 \pm 4.24$ & 0.098 \\
\hline $\mathrm{Ca}$ & $3159.20 \pm 1.41$ & $12337.20 \pm 7.07$ & $2568.20 \pm 12.73$ & $7745.20 \pm 6487.00$ & $2568.20 \pm 12.73$ & 0.067 \\
\hline $\mathrm{Ti}$ & $70308.70 \pm 2.12$ & $4444.50 \pm 12.02$ & $35372.20 \pm 2.83$ & $70308.70 \pm 2.12$ & $19905.10 \pm 21876.61$ & 0.003 \\
\hline V & $1277.60 \pm 2.12$ & $281.10 \pm 2.12$ & $660.90 \pm 8.49$ & $777.85 \pm 704.63$ & $660.90 \pm 8.49$ & 0.163 \\
\hline $\mathrm{Cr}$ & $550.40 \pm 20.51$ & $528.00 \pm 21.92$ & $279.00 \pm 0.71$ & $538.70 \pm 37.05$ & $279.00 \pm 0.71$ & 0.000 \\
\hline $\mathrm{Mn}$ & $3371.30 \pm 2.83$ & $451.40 \pm 4.24$ & $1728.90 \pm 6.36$ & $1908.85 \pm 2065.39$ & $1728.90 \pm 6.36$ & 0.169 \\
\hline $\mathrm{Fe}$ & $121923.60 \pm 31.11$ & $84729.80 \pm 4.95$ & $100948.90 \pm 8.49$ & $103335.95 \pm 26318.02$ & $100948.90 \pm 8.49$ & 0.169 \\
\hline Co & $260.70 \pm 4.95$ & $349.90 \pm 7.78$ & $383.00 \pm 39.03$ & $370.90 \pm 4.24$ & $416.60 \pm 8.49$ & 0.003 \\
\hline $\mathrm{Cu}$ & $370.30 \pm 2.12$ & $239.70 \pm 4.24$ & $295.40 \pm 2.12$ & $239.70 \pm 4.24$ & $295.40 \pm 2.12$ & 0.000 \\
\hline $\mathrm{Zn}$ & $220.20 \pm 4.95$ & $90.40 \pm 3.96$ & $136.60 \pm 4.24$ & $90.40 \pm 3.96$ & $136.60 \pm 4.24$ & 0.000 \\
\hline $\mathrm{Sr}$ & $89.70 \pm 10.61$ & $329.50 \pm 28.28$ & $71.50 \pm 2.83$ & $329.50 \pm 28.28$ & $71.50 \pm 2.83$ & 0.000 \\
\hline $\mathrm{Zr}$ & $2592.90 \pm 6.36$ & $223.80 \pm 4.95$ & $2261.30 \pm 8.49$ & $223.80 \pm 4.95$ & $2261.30 \pm 8.49$ & 0.000 \\
\hline Sn & $127.60 \pm 5.66$ & $127.60 \pm 5.66$ & $70.50 \pm 4.24$ & $113.00 \pm 3.54$ & $113.00 \pm 3.54$ & 0.000 \\
\hline $\mathrm{Pb}$ & $44.25 \pm 1.91$ & $44.10 \pm 0.71$ & $44.70 \pm 1.41$ & $45.95 \pm 2.12$ & $46.30 \pm 1.41$ & 0.574 \\
\hline $\mathrm{Bi}$ & $27.90 \pm 2.12$ & $34.30 \pm 2.12$ & $34.30 \pm 2.12$ & $31.50 \pm 1.41$ & $30.30 \pm 1.41$ & 0.071 \\
\hline Sc & $232.60 \pm 2.83$ & $218.35 \pm 0.49$ & $222.00 \pm 1.70$ & $219.75 \pm 1.48$ & $228.90 \pm 8.06$ & 0.057 \\
\hline $\mathrm{Ni}$ & $87.35 \pm 0.21$ & $86.10 \pm 0.71$ & $84.85 \pm 1.48$ & $85.75 \pm 1.34$ & $87.90 \pm 0.99$ & 0.149 \\
\hline $\mathrm{Cd}$ & $120.35 \pm 0.21$ & $91.35 \pm 0.21$ & $45.65 \pm 0.31$ & $98.35 \pm 0.21$ & $98.35 \pm 0.21$ & 0.001 \\
\hline $\mathrm{Au}$ & $14.70 \pm 0.14$ & $14.70 \pm 0.14$ & $13.75 \pm 0.21$ & $13.75 \pm 0.21$ & $15.55 \pm 0.35$ & 0.002 \\
\hline S & $489.25 \pm 1.91$ & $489.25 \pm 1.91$ & $472.80 \pm 2.83$ & $472.80 \pm 2.83$ & $506.80 \pm 8.49$ & 0.003 \\
\hline Y & $41.50 \pm 0.71$ & $41.50 \pm 0.71$ & $38.50 \pm 0.71$ & $38.50 \pm 0.71$ & $41.00 \pm 1.41$ & 0.036 \\
\hline
\end{tabular}

The above Table 2 showed the mean comparison of heavy metals in sediments. It showed that there is significant spatial variation in all the heavy metals except $\mathrm{Al}, \mathrm{P}, \mathrm{Cl}, \mathrm{K}, \mathrm{Ca}, \mathrm{V}, \mathrm{Mn}, \mathrm{Fe}, \mathrm{Pb}, \mathrm{Bi}, \mathrm{Sc}$ and $\mathrm{Ni}$. 
Table 3

Range and amount of elemental concentration in the sediment samples at Igbekebo $(\mathrm{mg} / \mathrm{kg})$ compound with other studies.

\begin{tabular}{|c|c|c|c|}
\hline Elements & Present study range (mean) & 1AEA - 457 & $\begin{array}{l}\text { East Attica, Greece } \\
\text { (Alexakis 2008)(mean) }\end{array}$ \\
\hline $\mathrm{Na}$ & $4551.20-6550.90(5287)$ & NA & 8500 \\
\hline $\mathrm{Mg}$ & 22752.60-25127.60(23636.8) & NA & 16083 \\
\hline $\mathrm{Al}$ & $2429.00-121552.80(54111)$ & 82660 & 36200 \\
\hline $\mathrm{Si}$ & $245744.90-346361.80(248121.4)$ & NA & NA \\
\hline$P$ & $117.90-460.40(308.4)$ & NA & NA \\
\hline $\mathrm{Cl}$ & $88.00-271.30(162.01)$ & NA & NA \\
\hline K & $1464.60-5222.40(2722.77)$ & NA & 7400 \\
\hline $\mathrm{Ca}$ & $2568.20-12337.20(5675.60)$ & NA & 122200 \\
\hline $\mathrm{Ti}$ & $4444.50-70308.70(40067.84)$ & NA & 2288 \\
\hline V & $281.10-1277.60(731.67)$ & NA & NA \\
\hline $\mathrm{Cr}$ & $279.00-550.40(435.02)$ & 144 & 285.8 \\
\hline $\mathrm{Mn}$ & 451.40-3371.30(1837.87) & 427 & 716 \\
\hline $\mathrm{Fe}$ & $84729.80-121923.60(102364.51)$ & 41,450 & 27,169 \\
\hline Zn & $90.40-220.20(134.84)$ & 425 & 169.98 \\
\hline $\mathrm{Sr}$ & $71.50-329.50(178.34)$ & 137 & 166 \\
\hline $\mathrm{Zr}$ & $223.80-2592.90(1512.62)$ & NA & 34.62 \\
\hline Sn & $70.50-127.60$ (110.34) & NA & NA \\
\hline $\mathrm{Pb}$ & $44.10-46.30(45.06)$ & 105 & 217.32 \\
\hline $\mathrm{Bi}$ & $27.90-34.30(31.66)$ & NA & NA \\
\hline Sc & $218.35-232.60$ (224.32) & NA & NA \\
\hline $\mathrm{Ni}$ & $84.85-87.90(86.39)$ & NA & NA \\
\hline $\mathrm{Cd}$ & $45.65-120(90.81)$ & NA & NA \\
\hline $\mathrm{Au}$ & $13.75-15.55(14.49)$ & NA & NA \\
\hline S & $472.80-506.80(486.18)$ & NA & NA \\
\hline Y & $38.50-41.50(40.20)$ & NA & NA \\
\hline Co & $260.70-416.60(356.22)$ & NA & NA \\
\hline $\mathrm{Cu}$ & $239.70-370.30(288.10)$ & 365 & 31.96 \\
\hline
\end{tabular}

Table 3 showed that sodium, magnesium and titanium concentrations in Igbekebo River sediment ranged from 4551.20 to $6550.90,22752.60$ to 25127.60 and 4444.50 to 70308.90 , respectively. With mean values of $2278785.45,23940.10$ and 37376.60 , respectively. Mean concentrations of sodium, magnesium and titanium were higher in Eastern Attica, Greece (Alexakis 2008). However, the mean value of sodium, magnesium and titanium could not be compared with the value of the IAEA, as they were not available at the time of the research. Chromium, manganese, iron and strontium concentrations ranged from 279.00 to $550.40,451.40$ to $3371.30,84729.80$ to 121923.60 , and 71.50 to 329.50 , respectively, with mean values of $414.70,1911.35,103326.70$ and 200.50 , respectively, which were higher than those of both the IAEA-457 and East Attica, Greece (Alexakis 2008). Potassium and calcium concentrations ranged from 1464.60 to 5222.40 . The mean potassium and calcium values were lower than those of East Attica, Greece (Alexakis 2008), but these mean potassium and calcium values could not be compared with the mean IAEA-457 because they are not available at the research point. Zirconium ranges from 223.80 to 2592.90 with a mean value of 1408.35 , which is also high as that of East Attica, Greece (Alexakis 2008).Aluminum and copper concentrations ranged from 2429.00 to 121552.80 and 239.70 to 370.30 , respectively, with mean values of 61990.90 and 305.00 , which were lower than the average value of IAEA-457 and higher than the mean value of East Attica, Greece (Alexakis 2008). Zinc and lead concentrations range from 90.40 to 220.20 and 44.10 to 46.30 , respectively, with mean values of 155.30 and 67.25 lower than the mean values of IAEA-457 and East Attica, Greece (Alexakis 2008). The order of the elemental concentration in the sediment sample is $\mathrm{Au}<\mathrm{Bi}<\mathrm{Y}<\mathrm{Pb}<\mathrm{Ni}<\mathrm{Sn}<\mathrm{Cl}<\mathrm{Sc}<\mathrm{Cu}<\mathrm{Cu}<\mathrm{Cr}<\mathrm{Cr}<\mathrm{Cr}<\mathrm{Cr}<\mathrm{V}<\mathrm{Cr}<\mathrm{Mn}<\mathrm{K}<\mathrm{Na}<\mathrm{Ca}<\mathrm{Mg}<\mathrm{Al}<\mathrm{Ti}<\mathrm{Fe}<\mathrm{Si}$. $\mathrm{High}$ concentrations of these elements in sediment could affect animals, insects and micro-organisms living in water. 
Table 4

Heavy metal concentration in both male and female frogs

\begin{tabular}{|c|c|c|c|}
\hline Heavy Metals & Male frog & Female frog & WHO Limit \\
\hline $\mathrm{Mg}$ & 2282 & 2831.6 & NA \\
\hline $\mathrm{Al}$ & 318.4 & 417.9 & NA \\
\hline Si & 2348.5 & 1832.5 & NA \\
\hline$P$ & 85.9 & 74.8 & NA \\
\hline $\mathrm{Cl}$ & 72.7 & 57.3 & NA \\
\hline K & 1219.2 & 1303.1 & NA \\
\hline $\mathrm{Ca}$ & 990.4 & 1006.8 & NA \\
\hline $\mathrm{Ti}$ & 1934.5 & 2122.1 & NA \\
\hline V & 89.3 & 107.2 & NA \\
\hline $\mathrm{Cr}$ & 110.5 & 132.2 & 0.07 \\
\hline $\mathrm{Mn}$ & 213.2 & 283.7 & 0.5 \\
\hline $\mathrm{Fe}$ & 45752 & 65035.9 & 0.5 \\
\hline Co & 88.9 & 101.1 & NA \\
\hline $\mathrm{Ni}$ & 64.3 & 63.6 & NA \\
\hline $\mathrm{Cu}$ & 61.1 & 102.4 & NA \\
\hline Zn & 81 & 63 & 5 \\
\hline $\mathrm{Zr}$ & 157.9 & 167.4 & NA \\
\hline $\mathrm{Cd}$ & 20.9 & 28.1 & NA \\
\hline $\mathrm{Pb}$ & 14.3 & 35.9 & 1 \\
\hline Sn & 20.8 & 22.2 & NA \\
\hline
\end{tabular}

During this study, male and a female frog were caught from oil producing community and both frogs were investigated for their elemental concentrations. The findings showed that in the samples high concentration elements in both frogs. Stolyar et al.2008 had also reported high concentration of elements in frogs living on the urban site in the Ukraine in comparisons between metal bioavailability in urban and rural frogs. Frogs are more sensitive to environmental toxins than other vertebrates because they do not allow foreign substances to be obstructed by egg membranes or skin (Duellman 1994; Snodgrass et al. 2003).

frogs have been used as bioindicators for pollution monitorisation, they have the proclivity to accumulate heavy metals (Berzins and Bundy 2002; Haywood et al. 2004; Simon et al. 2012). The consumption of frog is a result of Its availability, the taste for people and the need to meet the demand for protein are some of the key reasons for the annual consumption of billions of frogs. Frogs are also collected for leather production and souvenirs, pet trade and cultural purposes, including traditional medicine (Kusrini and Alford, 2006; Gonwouo and Rodel, 2008). From Table 4, Silicone (Si) had the concentration in both male frog and female while lead $(\mathrm{Pb})$ showed the least concentration in both frogs, The female frog accumulated higher concentrations of elements than the male frog. This split difference could be as a result of their genetic makeup. The results of the study can be compared to other studies (Zocche et al 2013; Stolyar et al.,2008) 
Table 5

Mean comparison of metals in male and female frogs

\begin{tabular}{|c|c|c|c|}
\hline & Male & Female & $\mathbf{P}$ \\
\hline $\mathrm{Mg}$ & $2282.00 \pm 10.00$ & $2831.60 \pm 500.00$ & 0.130 \\
\hline $\mathrm{Al}$ & $328.7 \pm 344.18$ & $417.90 \pm 179.00$ & 0.016 \\
\hline Si & $2348.57 \pm 348.50$ & $1832.50 \pm 500.00$ & 0.216 \\
\hline$P$ & $85.93 \pm 5.85$ & $74.80 \pm 10.00$ & 0.171 \\
\hline $\mathrm{Cl}$ & $72.73 \pm 2.65$ & $51.30 \pm 10.00$ & 0.023 \\
\hline K & $1252.60 \pm 270.65$ & $1303.10 \pm 300.00$ & 0.839 \\
\hline $\mathrm{Ca}$ & $990.53 \pm 90.20$ & $1006.80 \pm 100.00$ & 0.845 \\
\hline $\mathrm{Ti}$ & $1934.50 \pm 400.00$ & $2122.07 \pm 121.95$ & 0.481 \\
\hline V & $89.30 \pm 9.00$ & $107.20 \pm 10.00$ & 0.083 \\
\hline $\mathrm{Mn}$ & $213.20 \pm 13.20$ & $283.70 \pm 83.00$ & 0.220 \\
\hline $\mathrm{Fe}$ & $45752.00 \pm 752.00$ & $65035.90 \pm 5000.00$ & 0.003 \\
\hline $\mathrm{Cu}$ & $61.07 \pm 3.05$ & $102.40 \pm 10.00$ & 0.002 \\
\hline Zn & $81.00 \pm 3.00$ & $63.00 \pm 10.00$ & 0.040 \\
\hline $\mathrm{Zr}$ & $157.90 \pm 20.00$ & $167.40 \pm 10.00$ & 0.503 \\
\hline Sn & $20.80 \pm 4.00$ & $35.90 \pm 10.00$ & 0.072 \\
\hline $\mathrm{Cr}$ & $110.50 \pm 10.00$ & $132.20 \pm 10.00$ & 0.057 \\
\hline $\mathrm{Pb}$ & $14.30 \pm 4.00$ & $22.20 \pm 5.00$ & 0.099 \\
\hline Co & $88.90 \pm 8.90$ & $101.10 \pm 10.00$ & 0.190 \\
\hline $\mathrm{Ni}$ & $54.30 \pm 4.00$ & $63.60 \pm 10.00$ & 0.209 \\
\hline $\mathrm{Cd}$ & $20.90 \pm 10.00$ & $28.10 \pm 10.00$ & 0.166 \\
\hline
\end{tabular}

The mean comparison of metals in male and female frogs is shown in Table 5. There was a significant difference $(p<0.05)$ in the mean concentrations of Al, $\mathrm{Cl}, \mathrm{Fe}, \mathrm{Cu}$ and $\mathrm{Zn}$, while the other elements did not show any significant differences ( $p>0.05)$. In Al, Fe and $\mathrm{Cu}$, females had higher concentrations than males, while in $\mathrm{Cl}$ and $\mathrm{Zn}$, males had higher concentrations than females. 
Table 6

Interrelationship between metals in Frog

\begin{tabular}{|c|c|c|c|c|c|c|c|c|c|c|c|c|c|c|c|c|}
\hline & $\mathrm{Mg}$ & Al & Si & $\mathbf{P}$ & $\mathrm{Cl}$ & K & $\mathrm{Ca}$ & $\mathrm{Ti}$ & V & $\mathrm{Mn}$ & $\mathrm{Fe}$ & $\mathrm{Cu}$ & $\mathrm{Zn}$ & $\mathrm{Zr}$ & Sn & $\mathrm{Cr}$ \\
\hline $\mathrm{Mg}$ & 1 & 0.772 & 0.078 & 0.045 & -0.260 & 0.618 & 0.616 & 0.459 & $.880^{*}$ & $.986^{* *}$ & $.868^{*}$ & $.857^{*}$ & -0.184 & 0.554 & $.963^{* *}$ & $.865^{\star}$ \\
\hline $\mathrm{Al}$ & & 1 & -0.215 & -0.285 & -0.636 & 0.510 & 0.502 & 0.722 & $.944^{* *}$ & 0.739 & $.931^{\star *}$ & $.945^{\star *}$ & -0.571 & 0.718 & $.903^{*}$ & $.966^{* *}$ \\
\hline $\mathrm{Si}$ & & & 1 & $.996^{\star *}$ & $.885^{*}$ & 0.730 & 0.734 & 0.377 & 0.078 & 0.240 & -0.357 & -0.353 & $.918^{\star \star}$ & 0.461 & 0.043 & 0.005 \\
\hline$P$ & & & & 1 & $.919^{* \star}$ & 0.678 & 0.682 & 0.295 & 0.010 & 0.203 & -0.405 & -0.404 & $.947^{\star \star}$ & 0.383 & -0.011 & -0.064 \\
\hline $\mathrm{Cl}$ & & & & & 1 & 0.336 & 0.340 & -0.077 & -0.376 & -0.122 & -0.696 & -0.702 & $.997^{* *}$ & 0.001 & -0.367 & -0.445 \\
\hline K & & & & & & 1 & $.998^{\star \star}$ & $.830^{*}$ & 0.731 & 0.737 & 0.343 & 0.355 & 0.410 & $.903^{*}$ & 0.675 & 0.681 \\
\hline $\mathrm{Ca}$ & & & & & & & 1 & $.832^{*}$ & 0.730 & 0.736 & 0.340 & 0.352 & 0.414 & $.905^{*}$ & 0.674 & 0.680 \\
\hline $\mathrm{Ti}$ & & & & & & & & 1 & 0.797 & 0.543 & 0.463 & 0.493 & -0.013 & $.988^{\star *}$ & 0.650 & 0.784 \\
\hline V & & & & & & & & & 1 & $.889^{*}$ & $.881^{*}$ & $.891^{\star}$ & -0.298 & $.833^{*}$ & $.974^{\star *}$ & $.997^{\star \star}$ \\
\hline $\mathrm{Mn}$ & & & & & & & & & & 1 & 0.797 & 0.789 & -0.042 & 0.645 & $.955^{\star \star}$ & $.863^{*}$ \\
\hline $\mathrm{Fe}$ & & & & & & & & & & & 1 & $.999^{* *}$ & -0.636 & 0.487 & $.918^{* *}$ & $.905^{*}$ \\
\hline $\mathrm{Cu}$ & & & & & & & & & & & & 1 & -0.642 & 0.513 & $.919^{\star \star}$ & $.916^{*}$ \\
\hline $\mathrm{Zn}$ & & & & & & & & & & & & & 1 & 0.071 & -0.289 & -0.369 \\
\hline $\mathrm{Zr}$ & & & & & & & & & & & & & & 1 & 0.712 & 0.811 \\
\hline Sn & & & & & & & & & & & & & & & 1 & $.968^{\star \star}$ \\
\hline $\mathrm{Cr}$ & & & & & & & & & & & & & & & & 1 \\
\hline \multicolumn{17}{|l|}{$\mathrm{Pb}$} \\
\hline \multicolumn{17}{|l|}{ Co } \\
\hline \multicolumn{17}{|l|}{$\mathrm{Ni}$} \\
\hline \multicolumn{17}{|l|}{$\mathrm{Cd}$} \\
\hline *. Co & rrelati & $n$ is sign & ficant at & e 0.05 I & vel (2-tail & & & & & & & & & & & \\
\hline
\end{tabular}

Table 6 shows the interrelationship between metals in Frog. Most of the metals showed significantly high correlation with each other indicating similar sources. 
Table 7

Enrichment value using $\mathrm{Al}$ and $\mathrm{Si}$ as reference elements and Igeo accumulation in location $\mathrm{A}$ to $\mathrm{E}$

\begin{tabular}{|c|c|c|c|c|c|c|c|c|c|c|c|c|c|c|c|}
\hline & A & & & B & & & C & & & D & & & $E$ & & \\
\hline & Al & Si & Igeo & Al & Si & Igeo & Al & $\mathrm{Si}$ & Igeo & Al & $\mathrm{Si}$ & Igeo & Al & $\mathrm{Si}$ & Igeo \\
\hline $\mathrm{Al}$ & 1.00 & 0.01 & -2.18 & 1.00 & 0.04 & 0.02 & 1.00 & 0.01 & -2.30 & 1.00 & 0.02 & -0.70 & 1.00 & 0.01 & -2.30 \\
\hline $\mathrm{Si}$ & 143.63 & 1.00 & 4.98 & 27.11 & 1.00 & 4.78 & 110.87 & 1.00 & 4.49 & 47.92 & 1.00 & 4.88 & 110.87 & 1.00 & 4.49 \\
\hline$P$ & 1.99 & 0.01 & -1.19 & 0.11 & 0.00 & -3.15 & 1.60 & 0.01 & -1.63 & 0.44 & 0.01 & -1.88 & 1.60 & 0.01 & -1.63 \\
\hline $\mathrm{Cl}$ & 4.56 & 0.03 & 0.01 & 0.32 & 0.01 & -1.62 & 2.48 & 0.02 & -1.00 & 1.08 & 0.02 & -0.58 & 2.48 & 0.02 & -1.00 \\
\hline K & 0.17 & 0.00 & -4.77 & 0.13 & 0.00 & -2.93 & 0.22 & 0.00 & -4.48 & 0.14 & 0.00 & -3.58 & 0.22 & 0.00 & -4.48 \\
\hline $\mathrm{Ca}$ & 0.43 & 0.00 & -3.39 & 0.37 & 0.01 & -1.43 & 0.38 & 0.00 & -3.69 & 0.38 & 0.01 & -2.10 & 0.38 & 0.00 & -3.69 \\
\hline $\mathrm{Ti}$ & 46.27 & 0.32 & 3.35 & 0.64 & 0.02 & -0.63 & 25.32 & 0.23 & 2.36 & 16.53 & 0.34 & 3.35 & 14.25 & 0.13 & 1.53 \\
\hline V & 29.75 & 0.21 & 2.71 & 1.42 & 0.05 & 0.53 & 16.74 & 0.15 & 1.76 & 6.47 & 0.14 & 2.00 & 16.74 & 0.15 & 1.76 \\
\hline $\mathrm{Cr}$ & 18.51 & 0.13 & 2.03 & 3.86 & 0.14 & 1.97 & 10.21 & 0.09 & 1.05 & 6.47 & 0.14 & 2.00 & 10.21 & 0.09 & 1.05 \\
\hline $\mathrm{Mn}$ & 12.01 & 0.08 & 1.40 & 0.35 & 0.01 & -1.50 & 6.70 & 0.06 & 0.44 & 2.43 & 0.05 & 0.58 & 6.70 & 0.06 & 0.44 \\
\hline $\mathrm{Fe}$ & 7.82 & 0.05 & 0.78 & 1.18 & 0.04 & 0.26 & 7.04 & 0.06 & 0.51 & 2.37 & 0.05 & 0.55 & 7.04 & 0.06 & 0.51 \\
\hline Co & 41.54 & 0.29 & 3.19 & 12.12 & 0.45 & 3.62 & 66.39 & 0.60 & 3.75 & 21.11 & 0.44 & 3.70 & 72.21 & 0.65 & 3.87 \\
\hline $\mathrm{Cu}$ & 24.91 & 0.17 & 2.46 & 3.51 & 0.13 & 1.83 & 21.62 & 0.20 & 2.13 & 5.76 & 0.12 & 1.83 & 21.62 & 0.20 & 2.13 \\
\hline $\mathrm{Zn}$ & 7.02 & 0.05 & 0.63 & 0.63 & 0.02 & -0.66 & 4.74 & 0.04 & -0.06 & 1.03 & 0.02 & -0.66 & 4.74 & 0.04 & -0.06 \\
\hline $\mathrm{Sr}$ & 0.91 & 0.01 & -2.33 & 0.72 & 0.03 & -0.45 & 0.78 & 0.01 & -2.65 & 1.19 & 0.02 & -0.45 & 0.78 & 0.01 & -2.65 \\
\hline $\mathrm{Zr}$ & 49.06 & 0.34 & 3.43 & 0.92 & 0.03 & -0.10 & 46.55 & 0.42 & 3.24 & 1.51 & 0.03 & -0.10 & 46.55 & 0.42 & 3.24 \\
\hline Sn & 29.71 & 0.21 & 2.71 & 6.46 & 0.24 & 2.71 & 17.86 & 0.16 & 1.85 & 9.40 & 0.20 & 2.53 & 28.63 & 0.26 & 2.53 \\
\hline $\mathrm{Pb}$ & 6.70 & 0.05 & 0.56 & 1.45 & 0.05 & 0.56 & 7.36 & 0.07 & 0.58 & 2.48 & 0.05 & 0.62 & 7.62 & 0.07 & 0.63 \\
\hline Sc & 5.03 & 0.04 & 0.15 & 1.03 & 0.04 & 0.06 & 5.22 & 0.05 & 0.08 & 1.70 & 0.04 & 0.07 & 5.38 & 0.05 & 0.12 \\
\hline $\mathrm{Au}$ & 7.42 & 0.05 & 0.71 & 1.61 & 0.06 & 0.71 & 7.55 & 0.07 & 0.61 & 2.48 & 0.05 & 0.61 & 8.54 & 0.08 & 0.79 \\
\hline$Y$ & 4.83 & 0.03 & 0.09 & 1.05 & 0.04 & 0.09 & 4.88 & 0.04 & -0.02 & 1.60 & 0.03 & -0.02 & 5.19 & 0.05 & 0.07 \\
\hline $\mathrm{Cd}$ & 1214.41 & 8.46 & 8.06 & 200.41 & 0.003 & 7.67 & 501.15 & 4.52 & 6.67 & 354.44 & 7.40 & 7.77 & 1079.69 & 9.74 & 7.77 \\
\hline
\end{tabular}

From Table 7, location A: the geoaccumulation value as shown in Table 7; Al, P, K, Ca and Sr were unpolluted; Y, Au, Sc, Pb, Zn, Fe and Cl were slightly polluted; $\mathrm{Mn}$ was moderately polluted; $\mathrm{V}, \mathrm{Cr}, \mathrm{Cu}$ and $\mathrm{Sn}$ were moderately polluted; $\mathrm{Zr}$, Co and Ti were severely polluted; and $\mathrm{Si}$ was extremely polluted. Location $\mathrm{B} ; \mathrm{P}, \mathrm{Cl}, \mathrm{K}$, $\mathrm{Ca}, \mathrm{Ti}, \mathrm{Mn}, \mathrm{Zn}, \mathrm{Sr}$ and $\mathrm{Zr}$ were unpolluted; $\mathrm{Au}, \mathrm{Y}, \mathrm{Sc}, \mathrm{Pb}, \mathrm{V}$, Fe and $\mathrm{Al}$ were slightly polluted; $\mathrm{Cu}$ and $\mathrm{Cr}$ were moderately polluted; Sn was moderately polluted; Co was severely polluted; Si was severely polluted ,Cd was extremely polluted. Location C; Al, Y, Sr, Zn, Ca, P and Cl were unpolluted; Sc, Au, Mn, Fe, and Pb were slightly polluted; $\mathrm{Sn}, \mathrm{Cr}$ and $\mathrm{V}$ were moderately polluted; $\mathrm{Cu}$ and $\mathrm{Ti}$ were moderately polluted; $\mathrm{Co}$ and $\mathrm{Zr}$ were severely polluted; Si was severely polluted. Location D; Zr, Y, Sr, Zn, Ca, K, P, Cl and Al were unpolluted; Mn, Fe, Pb, Au and Sc were slightly polluted; $\mathrm{Cu}$, $\mathrm{Cr}$ and V were moderately polluted; Sn was moderately polluted; $\mathrm{Co}$ and Ti were severely polluted; $\mathrm{Si}$ was severely polluted. Location $\mathrm{E} ; \mathrm{P}, \mathrm{Al}, \mathrm{Cl}, \mathrm{K}, \mathrm{Ca}, \mathrm{Zn}$ and $\mathrm{Sr}$ were unpolluted; $\mathrm{Y}, \mathrm{Au}$, $\mathrm{Sc}, \mathrm{Pb}, \mathrm{Fe}$ and $\mathrm{Mn}$ were slightly polluted; $\mathrm{Cr}$, $\mathrm{V}$ and $\mathrm{Ti}$ were moderately polluted; $\mathrm{Sn}$ and $\mathrm{Cu}$ were moderately polluted; $\mathrm{Zr}$ and $\mathrm{Co}$ were severely polluted; $\mathrm{Si}$ was severely polluted. In this present study, the calculation of the enrichment factor was replaced by Al and Si.In the locations Cd was extremely polluted

The order of sediment enrichment in Location A when Al was used as a reference element was: $\mathrm{Cd}>\mathrm{Si}>\mathrm{Zr}>\mathrm{Ti}>\mathrm{Co}>\mathrm{V}>\mathrm{Sn}>\mathrm{Cu}>\mathrm{Cr}>\mathrm{Mn}>\mathrm{Fe}>\mathrm{Au}>\mathrm{Zn}>\mathrm{Pb}>$ $\mathrm{Sc}>\mathrm{Y}>\mathrm{Cl}>\mathrm{P}>\mathrm{Sr}>\mathrm{Ca}>\mathrm{K}$ as shown in Table 8. When $\mathrm{Si}$ was used as a reference element, the sediment enrichment order was: $\mathrm{Cd}>\mathrm{Zr}>\mathrm{Ti}>\mathrm{Co}>\mathrm{V}=\mathrm{Sn}>\mathrm{Cu}>$ $\mathrm{Cr}>\mathrm{Mn}>\mathrm{Fe}=\mathrm{Au}=\mathrm{Zn}=\mathrm{Pb}>\mathrm{Sc}=\mathrm{Y}=\mathrm{Cl}>\mathrm{P}=\mathrm{Al}=\mathrm{Sr}>\mathrm{Ca}>=\mathrm{K}$. In Location $\mathrm{B}$, the order of the enrichment value when $\mathrm{Al}$ was : $\mathrm{Cd}>\mathrm{Si}>\mathrm{Co}>\mathrm{Sn}>\mathrm{Cr}>\mathrm{Cu}>\mathrm{Au}>\mathrm{Pb}$ $>\mathrm{Fe}>\mathrm{V}>\mathrm{Y}>\mathrm{Sc}>\mathrm{Zr}>\mathrm{Sr}>\mathrm{Ti}>\mathrm{Zn}>\mathrm{Ca}>\mathrm{Mn}>\mathrm{Cl}>\mathrm{K}>\mathrm{P}$. When $\mathrm{Si}$ was used; $\mathrm{Sn}>\mathrm{Cu}>\mathrm{Cr}>\mathrm{Au}>\mathrm{Pb}=\mathrm{V}>\mathrm{Co}>\mathrm{Al}=\mathrm{Fe}=\mathrm{Sc}=\mathrm{Y}>\mathrm{Zr}=\mathrm{Sr}>\mathrm{Zn}=\mathrm{Ti}>\mathrm{Mn}=\mathrm{Cl}==\mathrm{Ca}>\mathrm{K}=$ $\mathrm{P}>\mathrm{Cd}$. In Location $\mathrm{C}$, the enrichment value when $\mathrm{Al}$ was : $\mathrm{Cd}>\mathrm{Si}>\mathrm{Co}>\mathrm{Zr}>\mathrm{Ti}>\mathrm{Cu}>\mathrm{Sn}>\mathrm{V}>\mathrm{Cr}>\mathrm{Au}>\mathrm{Pb}>\mathrm{Fe}>\mathrm{Mn}>\mathrm{Sc}>\mathrm{Y}>\mathrm{Zn}>\mathrm{Cl}>\mathrm{P}>\mathrm{Sr}>\mathrm{Ca}>\mathrm{K}$. when $\mathrm{Si}$ was used: $\mathrm{Cd}>\mathrm{Co}>\mathrm{Zr}>\mathrm{Ti}>\mathrm{Cu}>\mathrm{Sn}>\mathrm{V}>\mathrm{Cr}>\mathrm{Pb}=\mathrm{Fe}=\mathrm{Mn}=\mathrm{Sc}=\mathrm{Zn}=\mathrm{Y}>\mathrm{Cl}=\mathrm{Al}=\mathrm{Sr}=\mathrm{P}>\mathrm{K}=\mathrm{Ca}$. In Location $\mathrm{D}$, when Al was used the enrichment values were in order of: $\mathrm{Cd}>\mathrm{Si}>\mathrm{Co}>\mathrm{Ti}>\mathrm{Sn}>\mathrm{V}>\mathrm{Cr}>\mathrm{Cu}>\mathrm{Pb}>\mathrm{Au}>\mathrm{Mn}>\mathrm{Fe}>\mathrm{Sc}>\mathrm{Y}>\mathrm{Zr}>\mathrm{Sr}>\mathrm{Cl}>\mathrm{Zn}>\mathrm{P}>\mathrm{Ca}>\mathrm{K}$. When $\mathrm{Si}$ was used: $\mathrm{Cd}>\mathrm{Co}>\mathrm{Ti}>\mathrm{V}>\mathrm{Cu}>\mathrm{Cr}>\mathrm{Pb}=\mathrm{Au}=$ $\mathrm{Mn}=\mathrm{Fe}>\mathrm{Sc}>\mathrm{Zr}=\mathrm{Y}>\mathrm{Sn}=\mathrm{Sr}=\mathrm{Zn}=\mathrm{Cl}=\mathrm{Al}>\mathrm{P}=\mathrm{Ca}>\mathrm{K}$. In Location $\mathrm{E}$, when Al was used as reference element, the order of enrichment values were $: \mathrm{Cd}>\mathrm{Si}>\mathrm{Co}$ $>\mathrm{Zr}>\mathrm{Sn}>\mathrm{Cu}>\mathrm{V}>\mathrm{Ti}>\mathrm{Cr}>\mathrm{Au}>\mathrm{Pb}>\mathrm{Fe}>\mathrm{Mn}>\mathrm{Sc}>\mathrm{Y}>\mathrm{Zn}>\mathrm{Cl}>\mathrm{P}>\mathrm{Sr}>\mathrm{Ca}>\mathrm{K}$. When Si was used: $\mathrm{Cd}>\mathrm{Co}>\mathrm{Zr}>\mathrm{Sn}>\mathrm{Cu}>\mathrm{V}>\mathrm{Ti}>\mathrm{Cr}>\mathrm{Au}>\mathrm{Pb}>\mathrm{Fe}=\mathrm{Mn}>\mathrm{Sc}=\mathrm{Y}>$ $\mathrm{Zn}>\mathrm{Cl}>\mathrm{P}=\mathrm{Sr}=\mathrm{Al}>\mathrm{K}=\mathrm{Ca}$. Generally, It was observed that when Al gave higher values when used as reference element during computation of enrichment value when compared to that of $\mathrm{Si}$. 
Table 8

Bioaccumulation factor(BAF) target hazard quotient(THQ) and hazard index (HI) for both male frog and female frog

\begin{tabular}{|c|c|c|c|c|c|c|}
\hline Element & BAF male frog & BAF female frog & THQ male frog & THQ female frog & $\begin{array}{l}\text { RFD(USEPA } 2013 \\
\text { 2011) }\end{array}$ & RFD(RAIS2017) \\
\hline $\mathrm{Ti}$ & 0.48 & 0.053 & 0.00015 & 0.00016 & 4 & \\
\hline $\mathrm{Cr}$ & 0.254 & 0.304 & 0.01105 & 0.01322 & 0.003 & \\
\hline $\mathrm{Mn}$ & 0.116 & 0.154 & 0.0027 & 0.0036 & 0.024 & \\
\hline $\mathrm{Fe}$ & 0.447 & 0.635 & 0.0196 & 0.0279 & 0.7 & \\
\hline Co & 0.2496 & 0.284 & 0.00133 & 0.00152 & 0.02 & \\
\hline $\mathrm{Ni}$ & 0.744 & 0.736 & 0.000965 & 0.000954 & - & 0.02 \\
\hline $\mathrm{Cu}$ & 0.212 & 0.355 & 0.000494 & 0.000828 & 0.0371 & \\
\hline $\mathrm{Zn}$ & 0.601 & 0.467 & 0.00008 & 0.0000603 & 0.3 & \\
\hline $\mathrm{Al}$ & 0.059 & 0.0339 & 0.2388 & 0.3140 & 0.0004 & \\
\hline $\mathrm{Cd}$ & 0.20 & 0.790 & 0.0258 & 0.02187 & 0.001 & \\
\hline $\mathrm{Pb}$ & 0.32 & 0.797 & 0.00123 & 0.0007 & 0.0035 & \\
\hline V & 0.122 & 0.147 & 0.00531 & 0.00638 & - & 0.00504 \\
\hline $\mathrm{Zr}$ & 0.104 & 0.110 & - & - & - & - \\
\hline $\mathrm{Mg}$ & 0.097 & 0.01198 & - & - & - & - \\
\hline $\mathrm{Si}$ & 0.0095 & 0.0074 & - & - & - & - \\
\hline $\mathrm{P}$ & 0.279 & 0.243 & - & - & - & - \\
\hline $\mathrm{Cl}$ & 0.171 & 0.354 & - & - & - & - \\
\hline K & 0.45 & 0.48 & - & - & - & - \\
\hline $\mathrm{Ca}$ & 0.175 & 0.177 & - & - & - & - \\
\hline $\mathrm{HI}$ & & & 0.307509 & 0.391195 & & \\
\hline
\end{tabular}

From Table 8 , bioaccumulation factor in male frog was: $\mathrm{Cd}>\mathrm{Ni}>\mathrm{Zn}>\mathrm{Ti}>\mathrm{K}>\mathrm{Fe}>\mathrm{Pb}>\mathrm{P}>\mathrm{Cr}>\mathrm{Co}>\mathrm{Cu}>\mathrm{Ca}>\mathrm{Cl}>\mathrm{V}>\mathrm{Mn}>\mathrm{Zr}>\mathrm{Mg}>\mathrm{Al}>\mathrm{Si}$ while female frog : $\mathrm{Pb}>\mathrm{Cd}>\mathrm{Ni}>\mathrm{Fe}>\mathrm{K}>\mathrm{Zn}>\mathrm{Cu}>\mathrm{Cl}>\mathrm{Cr}>\mathrm{Co}>\mathrm{P}>\mathrm{Ca}>\mathrm{Mn}>\mathrm{V}>\mathrm{Mg}>\mathrm{Zr}>\mathrm{Ti}>\mathrm{Al}>\mathrm{Si}$. Cd had the highest bioaccumulation and Silicon had the lowest in male frog while $\mathrm{Pb}$ was the highest in female frog $\mathrm{In}$ totality , $\mathrm{Pb}$ was the highest bioaccumulation factor. The target hazard quotient as shown in Table 8 had decreasing order ; $\mathrm{Al}>\mathrm{Cd}>\mathrm{Fe}>\mathrm{Cr}>\mathrm{V}>\mathrm{Mn}>\mathrm{Co}>\mathrm{Pb}>\mathrm{Ni}>\mathrm{Cu}>\mathrm{Ti}>\mathrm{Zn}$ while female frog $: \mathrm{Al}>\mathrm{Fe}>\mathrm{Cd}>\mathrm{Cr}>\mathrm{V}>\mathrm{Mn}>\mathrm{Co}>\mathrm{Ni}>\mathrm{Cu}>\mathrm{Pb}>\mathrm{Ti}>\mathrm{Zn}$. Aluminum had the highest quotient value and Zinc had the lowest value in both the male and the female frog. The hazard index of the female frog was higher than the hazard index of the male frog as shown in Table 8. The trend obtained in this study can be compared to the values reported by stolyar et al 2008 .

\section{Conclusion}

This research has a significant effect on biodiversity and, in particular, human health, as human activities such as such as the deposition of waste in the river, fishing activities carried out with the use of toxic chemicals, it has been observed that lgbekebo is an oil-producing area, there is an oil spill on the river which contributes to the presence of a high concentration of heavy metals. As is well established in this work, concentration of elements in both male and female frogs was compared with the available WHO standard limits, which showed that the concentration of heavy metals in frog samples was significantly higher than available WHO limits, making frogs in the Igbekebo area harmful for consumption. It was also observed that Iron(Fe) was the highest concentration of $45752.0 \mathrm{mg} / \mathrm{kg}$ and $65035.9 \mathrm{mg} / \mathrm{kg}$ in male and female frogs, respectively, due to the natural occurrence of high concentrations of iron in water, the use of toxic chemicals for fishing and the high deposition of waste and metallic materials in the river. Furthermore, the results of heavy metals in sediment samples give convincing reasons for the high concentration of heavy metals in both male and female frogs. This study will support both the conservation authority and the consumption of these frog species, which are susceptible to pollution by heavy metals.

\section{Declarations}

Ethical Approval: The authors observed all ethics during the research and sought for the necessary approval

Consent to Participate: Not applicable

Consent to Publish:The authors give their consent to publish the manuscript 
Authors Contributions: Ogunjobi Adedayo Johnson initiated the work and designed the topic, Ediagbonya Thompson Faraday supervised the field work and the analytical work Adenikinji Charles Ademola, carried out the literature review,field work and the instrumental analyses,Ediagbonya Thompson Faraday,designed the paper,reproduced the tables and did the editorial work

Funding: Partial funding by the institution

Availability of data and materials: Not applicable

Conflict of interest: The authors declare that they have no conflict of interest

\section{References}

Abere SA, Lameed GA (2008). The medicinal utilization of snails in some selected States in Nigeria. In: Onkwelu, JC, Adekunle VAJ, Oke DO (eds.). Proceedings of the 1st National Conference of the Forests and Forest Products Society of Nigeria (FFPN) held at the Federal University of Technology, Akure, Ondo State, pp. 233-237.

Ademoroti CMO. (1996). Standard methods for water and effluents analysis: Ibadan Foludex press limited pp 29-118

Akpan, I. A., Wogar, G. S. I., Effiong, O. O. and Akpanenua, E. J. (2009). Growth performance of grasscutter fed diets treated with urea and urine solutions. Proc. Nig. Soc. for Anim. Prod. 34th Ann. Conf., 15th - 18th March, Uyo, Nigeria. pp 163 - 164.

Alexakis D (2008) Geochemistry of stream sediments as a tool for assessing contamination by Arsenic, Chromium and other toxic elements: East Attica region, Greece. Eur Water 21(22):57-72

Altherr, S., Goyenechea, A. and Schubert, D. (2011). Canapés to extinction- the international trade in frogs' legs and its ecological impact. A report by Pro Wildlife, Defenders of Wildlife and Animal Welfare Institute (eds.), Munich (Germany), Washington, D.C. (USA).

Angulo, A. (2008). Consumption of Andean frogs of the genus Telmatobius in Cusco, Peru: recommendations for their conservation. Traffic Bulletin 21: 95-97.

Avila, P.F., Ferreira da Silva, E. and Candeias, C. (2017), "Health risk assessment through consumption of vegetables rich in heavy elements: the case study of the surrounding villages from Panasqueira mine, Central Portugal”, Environmental Geochemistry and Health,39(3) 565-589.

Ballengee B, Sessions SK (2009) Explanation for missing limbs in deformed amphibians. J Exp Zool B Mol Dev Evol 312:770-77

Berzins D, Bundy K (2002) Bioaccumulation of lead in Xenopus laevis tadpoles from water and sediment. Environ Int 28:69-77

Burkhart JG et al (2000) Strategies for assessing the implications of malformed frogs for environmental health. Environ Health Perspect 108:83

Cohen MM (2001) Frog decline, frog malformations, and a comparison of frog and human health. Am J Med Genet 104:101-109

Duellman WE (1994) Biology of amphibians. JHU Press

DeForest, D.K., Brix, K.V. and Adams, W.J. (2007). "Assessing element bioaccumulation in aquatic environments: The inverse relationship between bioaccumulation factors, trophic transfer factors and exposure concentration", Aquatic Toxicology, 84 (2) 236-246

Ediagbonya TF \& Ayedun H (2018) Geochemistry of terrigenous sediments in surface water from ore and okitipupa southwest, Nigeria. Bangladesh Journal of Science and Industrial Research, 53(2), 145-154.

Ediagbonya TF and Balogun OT ( 2020) Potential risk assessment and spatial distribution of elemental concentrations in sediment Applied Water Science 10:176

Ediagbonya TF,Ogunjobi, JA and Olutayo, $\mathrm{OO}$ ( 2020) Effect of quarry activitieson selected biological resoures around quarry site within Onigambari forest plantation,Oyo State,Nigeria Environmental Geochemistry and Health 42 (4) :1-13

Efstathiadou C, Kostaropoulos IA, Loumbourdis NS (2007) Detoxification enzyme activities in the frog Rana ridibunda as a tool for evaluating the pollution of a freshwater ecosystem of Northern Greece with high concentrations of zinc and copper. Environ Bio Indic 2:60-70

Ergin M, Saydam CÖ. Baştürk Ö, Erdem E and Yörük R (1991) Heavy metal concentration in surface sediments from 2 inlets (Golden HornEstuary and İzmit Bay) of the north eastern Sea of Marmara. Chem. Geol., 91: 269-285.

FAO/WHO. (2010). Codex Alimentarius Commission Procedural Manual (19th Ed.). Rome, Italy: World Health Organization and Food and Agriculture Organization of the United Nations.

Fenoglio C, Albicini F, Milanesi G, Barni S (2011) Response of renal parenchyma and interstitium of Rana snk. esculenta to environmental pollution. Ecotoxicol Environ Saf 74:1381-1390

Gonwouo, L. N. and Rödel, M. O. (2008). The importance of frogs for the livelihoods of the Bakossi people around Mount Manengouba, Cameroon, with Special Consideration of the Hairy Frog, Trichobatrachus robustus. Salamandra 44: 23-34.

Page $12 / 14$ 
Hayes TB et al (2006) Pesticide mixtures, endocrine disruption, and amphibian declines: are we underestimating the impact? Environ Health Perspect 114:40 Haywood LK, Alexander GJ, Byrne MJ, Cukrowska E (2004) Xenopus laevis embryos and tadpoles as models for testing for pollution by zinc, copper, lead and cadmium. Afr Zool 39:163-174

He, F., Buoso, M.C., Burattini, E., Fazinic, S., Galassini, S., Haque, A.M.I., Jaksic, M., Moschini, G., 1993. Target preparation for trace element determination of biological materials using nuclear techniques. Nucl. Instrum. Meth. Phys. Res. Sect. A. 334, 238

Hazrat, A., ,Ezzat, K and Ikram I(2019) Environmental Chemistry and Ecotoxicology of Hazardous Heavy Metals: Environmental Persistence, Toxicity, and Bioaccumulation Hindawi Journal of Chemistry Volume 2019, Article ID 6730305, 14 pages

IAEA(International Atomic energy agency) (2000), Analytical quality control services, trace elements in soil

Irfan Zia Qureshi, Zeshan Kashif, Muhammad Zaffar Hashmi, Xiaomei Su, Riffat Naseem Malik, Kalim Ullah, Jinxing Hu, Muhammad Dawood, Assessment of heavy metals and metalloids in tissues of two frog species: Rana tigrina and Euphlyctis cyanophlyctis from industrial city Sialkot, PakistanJaved, M. and Usmani, N. (2013). Assessment of heavy element (Cu, Ni, Fe, Co, Mn, Cr, Zn) pollution in effluent dominated rivulet water and their effect on glycogen metabolism and histology of Mastacembelus armatus", SpringerPlus 2: (1) 390

Johansson, S.A., Campbell, J.L., Malmqvist, K.G. (Eds.), 1995. Particle-Induced X-ray Emission Spectrometry (PIXE). , 1st ed. John Wiley and Sons, New York, 133.

Kusrini, M. D. and Alford, R. A. (2006). Indonesia's exports of frogs' legs. TRAFFIC Bulletin 21 (1): 13-24.

Liao, C.M. and Ling, M.P. (2003), "Assessment of human health risks for arsenic bioaccumulation in tilapia (Oreochromis mossambicus) and large-scale mullet (Liza macrolepis) from blackfoot disease area in Taiwan”, Archives of Environmental Contamination and Toxicology Vol. 45 No. 2, pp. $264-272$.

Loska K, Cebula J, Pelczar J, Wiechula D, \& Kwapulinski J(1997). Use of enrichment, and contamination factors together with geoaccumulation indexes to evaluate the content of $\mathrm{Cd}, \mathrm{Cu}$, and $\mathrm{Ni}$ in the Bybnik water reservoir in Poland. Water Air Soil Pollution, 93, 347-365

Loumbourdis N, Kostaropoulos I, Theodoropoulou B, Kalmanti D (2007) Heavy metal accumulation and metallothionein concentration in the frog Rana ridibunda after exposure to chromium or a mixture of chromium and cadmium. Environ Pollut 145:787-792

Mohneke, M. (2011). (Un)sustainable use of frogs in West Africa and resulting consequences for the ecosystem. Dissertation, Humboldt University Berlin. Available at http://edoc.hu

Mohneke, M., Onadeko, A. B. and Rödel, M. O. (2009). Exploitation of Frogs a review with a focus on West Africa. Salamandra 45 (4): $193-202$

Muller G. (1969) Index of geoaccumulation in sediments of the Rhine River. Geology Journal, 2, 109-118

Niasse, M., Afouda, A. and Amani, A. (2004). Reducing West Africa's vulnerability to climate impacts on water resources, wetlands and desertification. Gland (Switzerland), IUCN, $67 \mathrm{pp}$.

Oguntona, T (1998). Green Leafy Vegetables. In: Osagie, A.U. and O.U. Eka (Eds.). Nutritional Quality of Plant Foods. Post Harvest Research Unit, Department of Biochemistry, University of Benin, Benin City, Nigeria, pp: 120-133. ISBN: 978-2120-02-2

Omonona, A. O., Ekpenko, V. (2011). Haematology and prevalence of blood parasites of the common frog (Rana temporaria) in the tropical environment, Journal of Veterinary Medicine and Animal Health Vol. 3(2), 14-20, June 2011.

Onadeko, A. B., Egonmwan, R. I. and Saliu, J. K. ( 2011). Edible Amphibian Species: Local Knowledge of their Consumption in Southwest Nigeria and their Nutritional Value, West African Journal of Applied Ecology, Vol. 19: 67-76.

RAIS, 2017. Risk exposure models for chemicals user's guide. Risk Assess. Inf. Syst. URL. https://rais.ornl.gov/tools/rais_chemical_risk_guide.html, Accessed date: 1 January 2017

Schiff, K. C., \& Weisberg, S. B. (1999). Iron as a reference element for determining trace metal enrichment in southern California coastal shelf sediments. Marine Environmental Research, 48, 161-176

Schuytema GS, Nebeker AV (1996) Amphibian toxicity data for water quality criteria chemicals. US Environmental Protection Agency, National Health and Environmental Effect Research Laboratory,

Simon E, Braun M, Tóthmérész B (2010) Non-destructivemethod of frog (Rana esculenta L.) skeleton elemental analysis used during environmental assessment. Water Air \& Soil Pollut 209:467-471

Simon E, Puky M, Braun M, Tóthmérész B (2012) Assessment of the effects of urbanization on trace elements of toe bones. Environ Monit Assess 184:57495754

Page $13 / 14$ 
Singh, A., Sharma, R. K., Agrawal, M., \& Marshall, F. M. (2010). Risk assessment of heavy metal toxicity through contaminated vegetables from waste water irrigated area of Varanasi, India. Tropical Ecology, 51(2), 375-387.

Snodgrass JW, Hopkins WA, Roe JH (2003) Relationships among developmental stage, metamorphic timing, and concentrations of elements in bullfrogs (Rana catesbeiana). Environ Toxicol Chem 22:

Stuart, S., Hoffman, M., Chanson, J., Cox, N., Berridge, R., Ramani, P., and Young, B. (2008). Threatened Amphibians of the World. Lynx Editions, Barcelona, 758 pp.

Subotic, S., Jeftic, Z.V., Spasic, S., Hegedis, A., Krpo- Cetkovic, J., Lenhardt, M., 2013. Distribution and accumulation of elements (As, Cu, Fe, Hg, Mn, and Zn) in tissues of fifish species from different trophic levels in the Danube River at the conflfluence with the Sava River (Serbia). Environ. Sci. Pollut. Res. 20 (8):53095317.

Sura P, Wróbel M, Bronowicka P (2006) Season dependent response of the Marsh frog (Rana ridibunda) to cadmium exposure. Folia Biol 54:159-165

Tchounwou PB, Yedjou, CG, Patlolla, AK, and Sutton DJ(2012) Heavy metals toxicity and the environment. EXS.101: $133-164$.

Teixeira R. D., Pereira Mello S. C. R. and Lima dos Santos C. A. M. (2001).The World Market of Frog Legs. FAO/Globefish Research Programme Vol. 86, Rome,1- $44 \mathrm{pp}$.

USEPA(2013):Reference dose (RfD): Description and use in health risk assessments,Background Document 1A, Integrated risk information system (IRIS); United States Environmental Protection Agency: Washington, DC, 15 March 2013; http://www epa.gov/iris/rfd.htm.

USEPA. (2015). Regional ScreeningLevel (RSL) Summary Table. United States Environmental Protection Agency.

Vitt, L. J., Caldwell, J. P (2009). Herpetology; An Introductory Biology of Amphibians and Reptiles 3rd Edition, Elsevier Academic Press 30 Coporate Drive, Suit 400, Burlington, MA 01803, USA, 697pp.

Waller, R. W. (2004). Status and Trends of Amphibian Declines and Extinctions Worldwide. Science 306: 1783-1786.

Warkentin, I. G., Bickford, D., Sodhi, N. S. and Corey, J. A. (2009). Eating frogs to extinction, Conservation Biology 23 (4): 1056-1059pp.

Zocche, J J., Damiani A P., Hainzenreder,G., Mendonc,R A, Peres, P B., dos Santosc, C E Debastiani,R., Diasc,J F and de Andrade V M (2013)Assessment of heavy metal content and DNA damage in Hypsiboas faber (anuran amphibian) in coal open-casting mine environmental toxicology and pharmacology 36 : 194-201

\section{Figures}

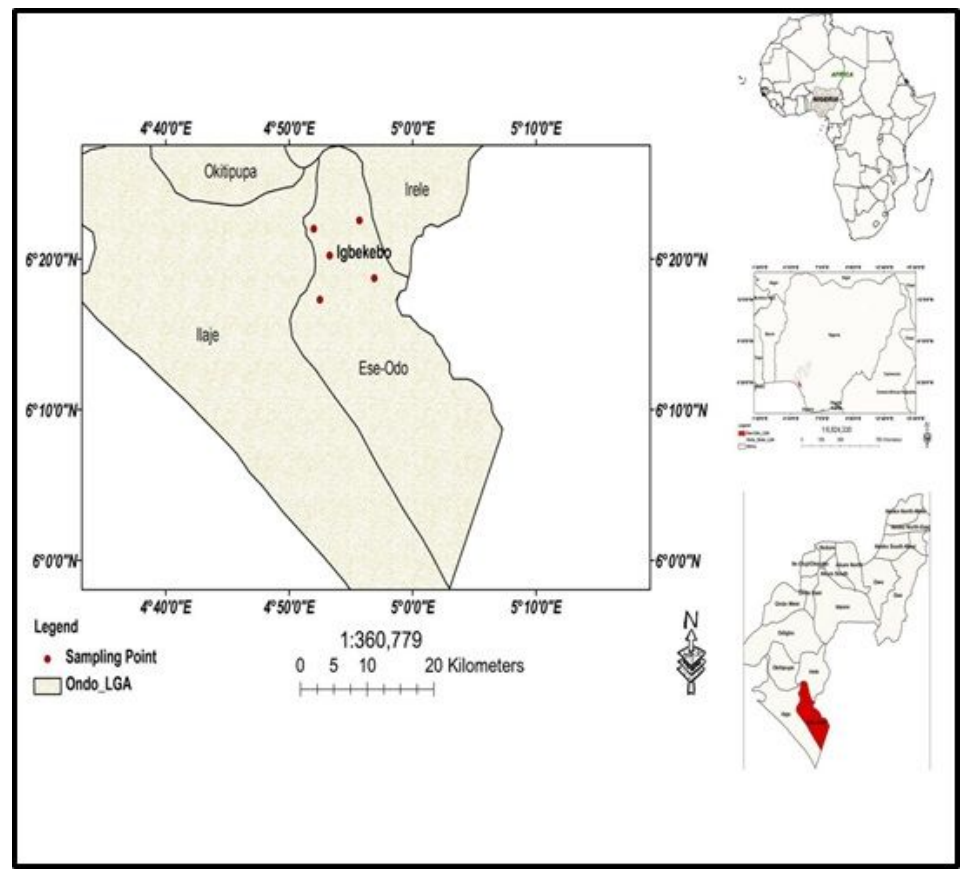

Figure 1

Map of Study Area 